\title{
Usefulness of transthoracic and transoesophageal echocardiography in recognition and management of cardiovascular injuries after blunt chest trauma
}

Fabio Chirillo, Oscar Totis, Antonio Cavarzerani, Andrea Bruni, Antonio Farnia, Mario Sarpellon, Paolo Ius, Carlo Valfrè, Paolo Stritoni

\begin{abstract}
Objective-To assess the diagnostic potential of transthoracic and transoesophageal echocardiography for the detection of traumatic cardiovascular injuries in patients suffering from severe blunt chest trauma.
\end{abstract}

Design-Prospective study over a three year period.

Setting-A regional cardiothoracic centre. Patients-134 consecutive patients (94 M/40 F; mean age 38 (SD 14) years) suffering from severe blunt chest trauma (injury severity score $33.5(18 \cdot 2))$. Most patients (89\%) were victims of motor vehicle accidents.

Evaluation-All patients underwent transthoracic and transoesophageal echocardiography within $8 \mathrm{~h}$ of admission. Aortography was performed in the first 20 patients and in a further five equivocal cases.

Results-Transthoracic echocardiography provided suboptimal images in 83 patients, detecting three aortic ruptures, 28 pericardial effusions (one cardiac tamponade), 35 left pleural effusions, and 15 myocardial contusions. Transoesophageal echocardiography was feasible in 131 patients and detected 14 aortic ruptures $(13$ at the isthmus), 40 pericardial effusions, 51 left pleural effusions, 34 periaortic haematomas, 45 myocardial contusions, right atrial laceration in one patient with cardiac tamponade, one tricuspid valve rupture, and one severe mitral regurgitation caused by annular disruption. For the detection of aortic rupture transoesophageal echocardiography showed 93\% sensitivity, $98 \%$ specificity, and $98 \%$ accuracy. Time to surgery was significantly shorter (30 (12) v 71 (21) min; $P<$ 0.05 ) for patients operated on only on the basis of transoesophageal echocardiographic findings.

Conclusions-Transthoracic echocardiography has low diagnostic yield in severe blunt chest trauma, while transoesophageal echocardiography provides accurate diagnosis in a short time at the bedside, is inexpensive, minimally invasive, and does not interfere with other diagnostic or therapeutic procedures.

(Heart 1996;75:301-306)

Keywords: blunt chest trauma; traumatic cardiovascular injuries; echocardiography
About $10 \%$ of victims of major trauma sustain cardiac or aortic lesions. ${ }^{1}$ Although the vast majority of these individuals die at the scene, ${ }^{2}$ an increasing number of patients with previously lethal injuries arrive at a treatment facility because of improvements in prehospital care, more aggressive resuscitation in the field, and rapid transportation to appropriate units. ${ }^{3}$ The identification of cardiovascular injury in these patients is often difficult because of severe associated lesions which are more apparent and require prompt treatment. ${ }^{4}$ The diagnostic value of routine techniques for the detection of traumatic cardiovascular injuries is limited ${ }^{56}$ and the accuracy of more refined techniques, such as computed tomography and magnetic resonance imaging, has been questioned..$^{7-9}$

Aortography is still considered to be the gold standard technique for the detection of traumatic aortic rupture, ${ }^{1011}$ but it cannot be always performed on an emergency basis, has the complications involved in an invasive technique, ${ }^{12}$ does not identify myocardial or valvar lesions, and has a low diagnostic yield and very high cost when performed on all trauma patients.

Transthoracic echocardiography has been used in previous studies ${ }^{13-15}$ on patients suffering from chest trauma: it proved helpful in detecting myocardial contusion, whereas no data have been reported on identification of aortic rupture.

Transoesophageal echocardiography overcomes most of the technical limitations of transthoracic echocardiography and provides excellent visualisation of the heart and almost the entire thoracic aorta in nearly all patients. ${ }^{1617}$ The aim of this study was to assess the diagnostic potential of transthoracic and transoesophageal echocardiography for the detection of traumatic cardiovascular lesions and to evaluate the impact of transoesophageal echocardiographic findings on the management of patients following severe blunt chest trauma.

\section{Methods}

Between September 1991 and September 1994134 consecutive patients (94 men, 40 women) aged 15-78 years (mean 38 (SD 14) years) admitted to our hospital following severe blunt chest trauma were prospectively evaluated for presence of cardiac injury and aortic rupture (table 1). Patients were entered into the study who were found to have haemothorax, pneumothorax, pulmonary contusion, fracture of the first two or multiple 
Table 1 Clinical characteristics of 134 patients with blunt chest trauma evaluated for cardiovascular injury

\begin{tabular}{ll}
\hline Sex M/F & $94 / 40$ \\
Age (years) & $38($ SD 14) \\
Mechanism of injury, $\mathrm{n}(\%)$ & \\
Drivers & $54(40)$ \\
Occupants & $33(25)$ \\
Pedestrians struck by car & $14(10 \cdot 5)$ \\
(Motor)cyclists & $18(13 \cdot 5)$ \\
Fall from height & $12(9)$ \\
Animal kicks & $3(2)$ \\
Injury severity score & $33 \cdot 5(\mathrm{SD} 18 \cdot 2)$ \\
Associated injuries, $\mathrm{n}(\%)$ & $86(64)$ \\
Cranial & $38(28)$ \\
Abdominal & $41(30 \cdot 5)$ \\
Pelvic & $16(2)$ \\
Major extremity fractures & $78(58)$ \\
Emergency surgical procedures, $\mathrm{n}$ & 145 \\
Neurosurgical & $15(11)$ \\
Abdominal & $35(26)$ \\
Urological & $7(5)$ \\
Orthopaedic & $55(41)$ \\
Thoracic (non-cardiovascular) & $19(14)$ \\
Cardiovascular & $15(11)$ \\
Chest film suggestive for aortic rupture, & \\
n (\%) & $65(48 \cdot 5)$ \\
Death, $\mathrm{n}(\%)$ & $19(14)$ \\
\hline
\end{tabular}

ribs, or to have had a significant field evidence of high energy transfer such as falls of more than 4 metres and automobile accidents with structural intrusion, extrication difficulties, passenger ejection, or death at the scene.

Motor vehicle accidents were the cause of injury in most patients. The injury severity score $^{1819}$ for the entire group was 33.5 (SD $18 \cdot 2)$. Most patients (64\%) experienced associated cranial, abdominal and pelvic injury, often requiring emergency surgery. Ninety seven patients arrived at our institution directly from the accident scene, and 37 were transferred from other hospitals. There were 19 deaths: five from head trauma, six from haemorragic shock, five from multisystem organ failure, and three from aortic rupture. In all but the latter three patients necropsy ruled out major cardiac injury and aortic rupture.

Eighty patients were evaluated while endotracheally intubated; 35 had chest tubes, 21 abdominal tubes, and five had subcutaneous emphysema.

Supine anteroposterior chest plain $x$ ray film was obtained in all patients at admission: mediastinal widening, a blurred aortic knob, apical capping of the lung, rightward displacement of the trachea, and depression of the left main bronchus were considered findings suggestive of aortic rupture. Chest $x$ ray was repeated in the erect position before discharge from the hospital.

Transthoracic and transoesophageal echocardiography were performed in all patients within $8 \mathrm{~h}$ of admission. Thirty seven echocardiographic studies were done while patients were undergoing other diagnostic or therapeutic procedures: peritoneal lavage (7), drainage of pneumothorax or haemothorax (10), reduction of extremity fractures (8), or packing of abdominal lesions (12). Echocardiographic studies were carried on in the operating room (20), intensive care unit (85), $x$ ray department (8), and emergency room (20).

Studies were performed by expert cardiologists using $2.5 \mathrm{MHz}$ and $3.5 \mathrm{MHz}$ transthoracic probes and a transoesophageal 5 $\mathrm{MHz}$ single plane probe mounted at the end of a $100 \mathrm{~cm}$ gastroscope and attached to a Sonos 1000 Hewlett-Packard ultrasound system. Introduction of the probe was accomplished after the administration of intravenous droperidole or morphine, when necessary, for sedation.

Cross sectional echocardiography identified myocardial contusion in presence of hypokinesis, akinesis, or dyskinesis of any segment of the left ventricle or right ventricular free wall. ${ }^{20}$ Patients with myocardial contusion underwent a control transthoracic echocardiogram before discharge. Pericardial and pleural effusion were diagnosed when an echo-free space was seen between the visceral and parietal pericardial and pleural echoes in all imaging planes. Periaortic haematoma was diagnosed when a bright echo density was seen around the aorta. Colour Doppler interrogation of the septa and valves identified intracardiac shunts or valvar regurgitation according to standard criteria. ${ }^{21} 22$ Aortic rupture was diagnosed when two or more of the following echographic criteria were met: (1) abrupt and discrete change in aortic diameter (that is, normal diameter proximal and distal to the site of rupture which exhibited widened lumen); (2) presence of one or more consistent linear echoes indicative of transection flaps dividing the aortic lumen into two or more lumina; (3) focal but complete intimal and medial disruption with formation of a pseudoaneurysmatic cavity; (4) colour Doppler identification of a pseudocoarctation pattern (that is, flow acceleration through the injured aortic segment); (5) periaortic haematoma.

Findings on echocardiography were compared with aortography in 25 patients, surgery in 15 patients, and necropsy in 19 cases. Patients with transoesophageal echocardiography negative for aortic rupture and repeat chest $x$ ray negative for widened mediastinum were considered free from aortic injury.

\section{STATISTICAL ANALYSIS}

Continuous values are expressed as means (SD). The sensitivity (true positives divided by true positives plus false negatives), specificity (true negatives divided by true negatives plus false positives), positive predictive value (true positives divided by true positives plus false positives), negative predictive value (true negatives divided by true negatives plus false negatives) and accuracy (true positives plus true negatives divided by total number of examinations performed) of transoesophageal echocardiography for aortic rupture were calculated. A $P$ value $<0.05$ was considered statistically significant.

\section{Results}

Transthoracic echocardiography provided suboptimal images of the heart and the aorta in 83 patients. Reasons were: mechanical ventilation (58), obesity (8), subcutaneous emphysema (5), chest or abdominal tubes (7). In five patients transthoracic studies were not performed in order to avoid contamination of sterile instrumentation used for other ongoing diagnostic and therapeutic procedures. 
Table 2 Findings on transthoracic and transoesophageal echocardiography in 134 patients suffering from blunt chest trauma

\begin{tabular}{lll}
\hline & $T T E, n(\%)$ & $T O E, n(\%)$ \\
\hline Feasibility & $51(38)$ & $131(98)$ \\
Aortic rupture & $3(6)$ & $14(10.5)$ \\
Myocardial contusion & $15(21 \cdot 5)$ & $45(34)$ \\
Valvar lesions & 0 & $2(1.5)$ \\
Periaortic haematoma & $5(10)$ & $34(26)$ \\
Pericardial effusion & $28(55)$ & $40(30.5)$ \\
Left pleural effusion & $35(69)$ & $51(39)$ \\
\hline
\end{tabular}

TTE, transthoracic echocardiography; TOE, transoesophageal echocardiography.

Findings on transthoracic echocardiography are shown in table 2 . In two patients with aortic rupture at the isthmus a pseudocoarctation pattern was noted. There were four false positives on transthoracic echocardiography for the identification of aortic rupture.
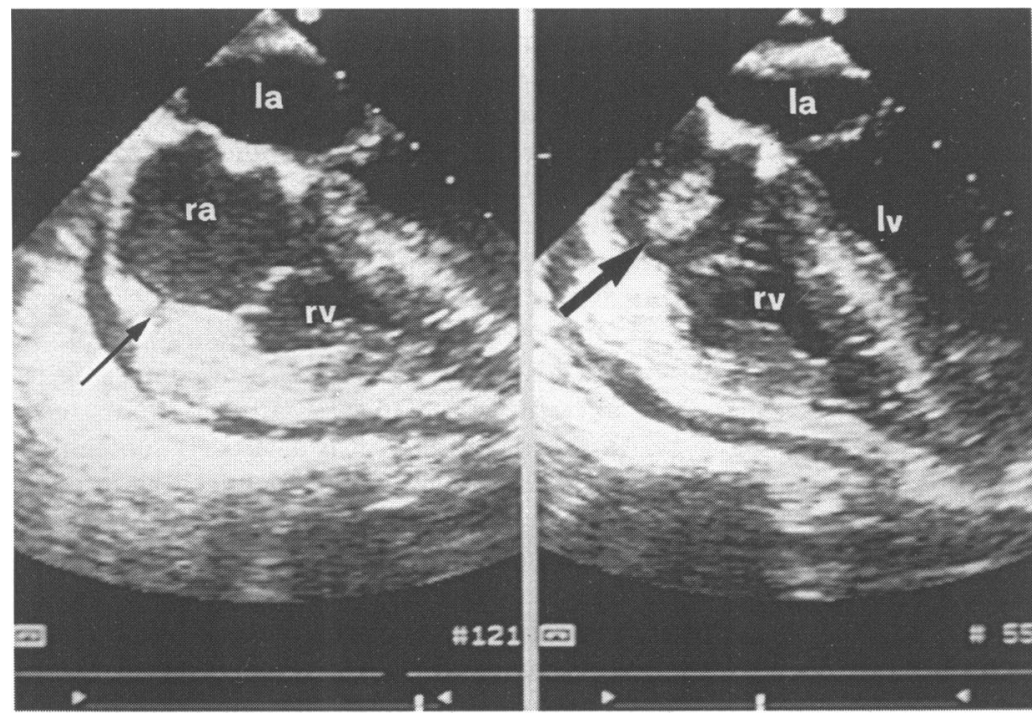

Figure 1 Transoesophageal basal four chamber view taken in a patient with cardiac tamponade following a horse kick. (Left) The arrow indicates a small fissuring on the right atrial wall. There is pericardial effusion and the right ventricle looks compressed. (Right) $A$ portion of the torn right atrial wall (arrow) is seen floating within the right atrium just above the tricuspid valve (la, left atrium; lv, left ventricle; ra, right atrium; rv, right ventricle).
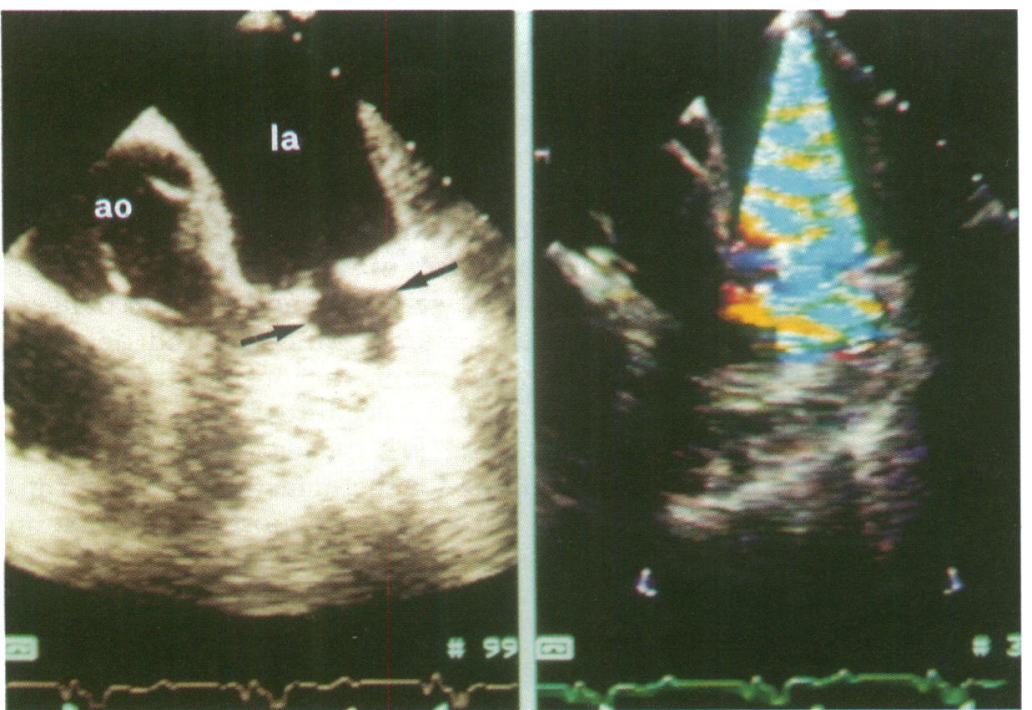

Figure 2 Transoesophageal basal two chamber view demonstrating a large tear in the anterior mitral annulus (arrows). In the right panel colour flow imaging shows massive paravalvar regurgitation (ao, aorta; la, left atrium).
Oesophageal intubation for transoesophageal echocardiography was impossible in three patients (one had tracheostomy after laryngectomy and two had fracture of the cervical spine with initial neurological deficit). Transoesophageal echocardiography detected 40 pericardial effusions, 51 left pleural effusions (five were large and undetected by chest $x$ ray, and were drained after transoesophageal study), 34 periaortic haematomas, 45 myocardial contusions (eight patients had left ventricular ejection fraction $<35 \%$ ). Transoesophageal echocardiography identified one tricuspid valve rupture, laceration of the right atrial lateral wall in one patient with cardiac tamponade (fig 1), and one severe mitral regurgitation caused by disruption of the anterior anulus (fig 2). These three patients were successfully operated on in the emergency department. Incidental findings on transoesophageal echocardiography were: one cor triatriatum, one mitral stenosis, and eight aneurysms of the atrial septum (four with mild left to right shunts).

Sixty five patients had suspected rupture of the aorta on chest $x$ ray. Confirmatory aortography was performed in the first 20 cases (to complete the learning curve), and in another five cases with equivocal transoesophageal echocardiographic findings. Transoesophageal echocardiography disclosed 14 aortic ruptures (13 at the isthmus) (fig 3); there were two false positives (due to reverberations in the ascending aorta) and one false negative (sensitivity $93 \%$, specificity $98 \%$, positive predictive value $87 \%$, negative predictive value $99 \%$, accuracy $98 \%$ ). This latter patient had pneumomediastinum caused by laceration of the left mainstem bronchus following a fall from a $5 \mathrm{~m}$ height. Transoesophageal study provided poor visualisation of the entire thoracic aorta due to the presence of air in the mediastinum. The study was repeated after left pneumectomy and detected rupture of the distal ascending aorta.

Time to surgery was significantly longer for patients undergoing aortography (71 (21) min $v 30$ (12) min; $P<0.05)$. One patient died during confirmatory aortography and another died during transfer to the operative room soon after transoesophageal echocardiographic evaluation. Twelve patients underwent reparation of the aortic rupture (fig 4) in the emergency department. There was one operative death from disseminated intravascular coagulation; 11 patients were discharged in good general condition. Comparison with surgical and necropsy findings showed that transoesophageal echocardiography was fully diagnostic in 11 cases. It could not identify one case of avulsion of the innominate artery concomitant to injury of the ascending aorta, one of avulsion of the left subclavian artery concomitant to aortic transection at the isthmus, and one of retrograde extension of the isthmic transection to the distal arch. In this patient surgeons had serious difficulties in approaching the arch rupture through a left thoracotomy, but operation was carried out successfully.

Repeat echo showed improvement in left ventricular systolic function in all patients with 


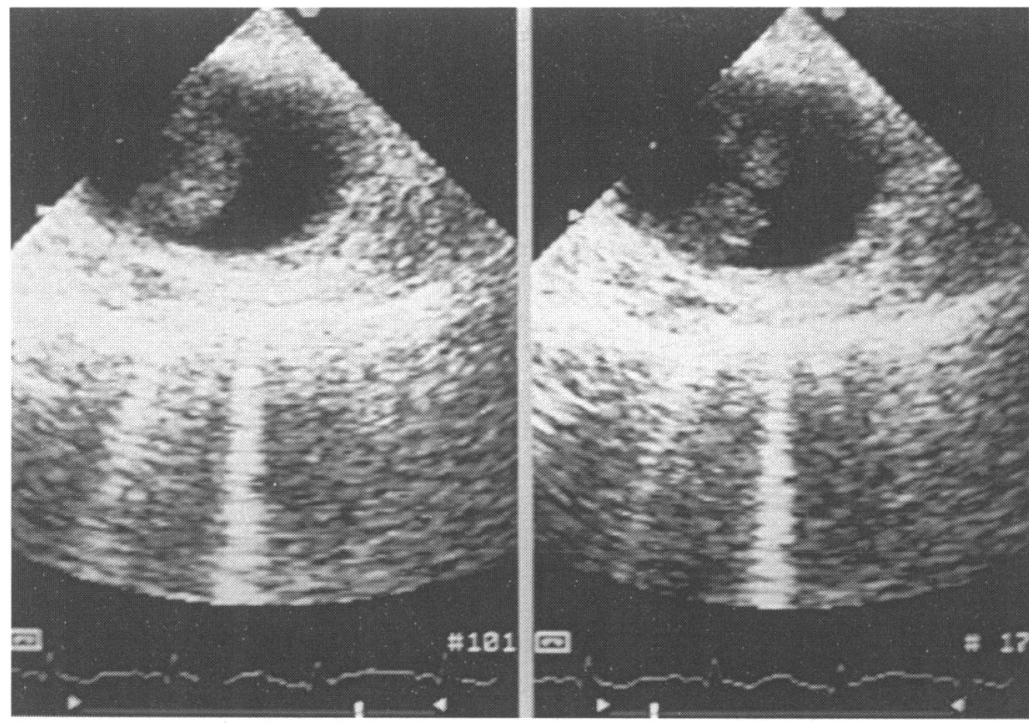

Figure 3 Traumatic aortic rupture at the isthmus. Transoesophageal scanning shows the presence of an echolucent structure presenting a "valve-like" movement (left is a diastolic frame, right a systolic frame). This aspect corresponded to the adventitia and periadventitial tissues invaginated into the aortic lumen through the traumatic tear

\section{4 \\ CHEST TRAUMA}

\section{CARDIOVASCULAR INJURIES}

\section{5}

\section{EMERGENCY SURGERY}

14

ALIVE

Figure 4 Outcome of 134 patients with severe blunt chest trauma. myocardial contusion and normalisation of all segmental abnormalities in 38 patients.

Repeat $x$ ray chest film did not show any significant enlargement of the mediastinum in patients with transoesophageal echocardiography negative for aortic rupture.

\section{Discussion}

The evaluation of patients suffering from blunt chest trauma for the detection of cardiovascular injury is challenging because most cardiovascular lesions occur in a milieu of multisystem trauma and often go undetected. In these patients the diagnostic value of routine techniques is limited because the electrocardiographic changes are non-specific and a chest film cannot be always performed in the upright position, resulting in uncertainties over mediastinal dimensions and structure. ${ }^{23-25}$ The portable chest radiograph is frequently compromised by limited exposure capability, expiratory views, patient motion, difficult patient positioning, and magnification and distortion of the mediastinum in the supine position. Moreover actual widening of the mediastinum may result from chronic anatomical changes such as enlargement of the thoracic aorta secondary to hypertension or atherosclerosis and thoracic neoplasms. In our experience an initial emergency room chest radiograph was obtainable in most patients only in the supine position because of the severity of trauma. Most of the abnormalities observed on the initial radiograph disappeared on the erect chest $x$ ray obtained before discharge from the hospital.

Transthoracic echocardiography provided suboptimal images in $62 \%$ of patients and the bad quality of the images obtained was the main cause of the low sensitivity of transthoracic echocardiography, especially when aortic rupture in the region of the isthmus (poorly visualised by the suprasternal notch) is searched for.

In the light of our experience we do not recommend transthoracic echocardiography as a routine technique in the evaluation of blunt chest trauma, either for the identification of aortic injury or for myocardial contusion. The use of this technique would consume time and increase the cost of management of trauma patients with low clinical yield. The only rather specific sign for isthmic transection on transthoracic echocardiography might be the presence of a pseudocoarctation pattern (peak transisthmic gradient $>20 \mathrm{~mm} \mathrm{Hg}$ ).

Transoesophageal echocardiography offers several advantages over other imaging methods currently used in evaluating patients with chest trauma. Firstly, it is at most a semi-invasive procedure which has been shown to be safe when used by experienced personnel. Potential complications may occur in patients with traumatic oesophageal and gastric lesions, since the probe is not provided with a fibreoptic capability. Excessive neck and head movements to facilitate oesophageal intubation might be harmful in patients who may have injuries to the cervical spine. Patients with suspected aortic injury should be heavily sedated to avoid any increase in blood pressure which may precipitate aortic rupture. ${ }^{26}$ Notwithstanding these potential risks, morbidity and mortality of transoesophageal studies were nil, as in previous studies. ${ }^{2728}$

Secondly, transoesophageal echocardiography can be performed quickly (since it does not require the assembly of an on-call radiology team), at the bedside (avoiding transportation of patients, which it is often difficult for the presence of mechanical ventilation, orthopaedic devices, etc, and may be dangerous in such unstable patients), and does not interfere with other diagnostic, therapeutic, and resuscitative procedures.

Thirdly, transoesophageal echocardiography is accurate for the detection of aortic rupture and provides diagnostic information about possible myocardial and cardiac valvar injury as well as about presence and magnitude of pericardial and left pleural effusion.

Many different criteria for the diagnosis of traumatic aortic rupture using transoesophageal echocardiography have been suggested. ${ }^{28-32}$ The inclusion of periaortic haematoma among our diagnostic criteria for 
aortic rupture may appear questionable. In fact Sandor ${ }^{33}$ reported that blood in the mediastinum was secondary to aortic rupture in only $12.5 \%$ of trauma patients and to nonaortic bleeding in $87.5 \%$. Other sources of periaortic haematoma include tearing of mediastinal veins, rib fractures, sternal fractures, injury to brachiocephalic vessels, and caudal dissection of blood from cervical injuries. ${ }^{34}$ Moreover Woodring et al $^{35}$ have reported nine cases of mediastinal widening associated with fractures of the cervical, thoracic, and lumbar spine. Such lesions were found in five patients in the present series. Periaortic haematoma therefore has poor specificity as a sign of aortic rupture following blunt chest trauma. However, it should be emphasised that in the present study it was not included among the diagnostic criteria on its own, and it presented $100 \%$ sensitivity. This may partly be dependent on the absence of patients with intimal injury $^{36}$ (that is, subclinical traumatic aortic injury which may occur without extravasation of blood from the aorta).

Limitations of transoesophageal echocardiography include: absent visualisation of the distal ascending aorta and proximal arch because of the interposition of the air filled trachea and left main bronchus; poor visualisation of the epiaortic vessels and low resolution in presence of pneumomediastinum and pneumopericardium. ${ }^{16}$ Biplane $^{17}$ and omniplane transoesophageal probes ${ }^{37}$ can provide better visualisation of the ascending aorta, but do not allow detailed analysis of the brachiocephalic vessels and cannot eliminate interference from air in the airways or in the mediastinum.

It is therefore clear that avulsion of the brachiocephalic vessels is not amenable to transoesophageal echocardiographic diagnosis, as are other conditions such as rupture of the distal ascending aorta and proximal arch, injury of the abdominal aorta, or avulsion of thoracic and abdominal aortic collaterals.

Previous studies ${ }^{30-32}$ dealing with smaller series of patients have reported different values of accuracy for detection of aortic rupture by transoesophageal echocardiography.

In our series one false negative and two false positives occurred at the beginning of our experience. Later on in the study both cardiologists and surgeons became confident with transoesophageal echocardiographic images and seven patients were operated upon without aortography. This underscores the accuracy of the technique in evaluating aortic rupture and the need of a learning curve, even for experienced echocardiographers. Experience is needed to select the views, to optimise partial and total gain (to avoid sonographic artefacts) and in the interpretation of images. ${ }^{29}$ If operators are not sufficiently skilled we recommend sending patients for angiography in the presence of dubious findings. This will undoubtedly increase initial cost, but will serve to complete the learning curve and ultimately reduce cost and increase diagnostic yielding in a near future. ${ }^{38}$

Time to surgery is crucial in acute aortic pathology. In the study by Smith et al ${ }^{28}$ all patients underwent both transoesophageal echocardiography and aortography with a mean 105 (56) min interval before achieving definite diagnosis. After the death of one patient during confirmatory aortography, we decided that was ethically correct to send patients with transoesophageal echocardiographic evidence of aortic rupture to operation without confirmatory aortography. Aortography was performed subsequently in only five patients with suspected involvement of the ascending aorta and arch vessels. This reduces the ability of the present study to assess the diagnostic accuracy of transoesophageal echocardiography in the diagnosis of traumatic aortic rupture, because this aim could only be properly explored by a comparison with aortography, so far considered the gold standard technique. This was carried out in the study by Smith et $a .^{28}$ Their reported sensitivity $(100 \%)$ and positive predictive value $(99 \%)$ were higher than in the present study. This may be because all the ruptures were localised at the isthmus (clearly visualised by transoesophageal imaging using single plane technology) and because no injuries of the arch vessels were reported. An interesting finding which emerges from comparative studies on transoesophageal echocardiography and aortography ${ }^{2836}$ is that this latter gold standard technique also has false negatives (incomplete aortic rupture, thrombosis of the pouch, or technical deficiencies such as poor opacification or inadequate number of views) and false positives (ductal diverticula or atheromatous aorta).

In the evaluation of aortic injury transoesophageal echocardiography should be considered not as an alternative, but as a complement to angiography. Transoesophageal echocardiography might be a means of selecting patients who must proceed directly to surgery (unequivocal evidence of aortic rupture), who must undergo aortography (periaortic haematoma and otherwise unexplained mediastinal widening), and who may benefit from aortography (patients with negative transoesophageal echocardiography). This latter policy will pick up any false negatives and also the patients with ascending aorta, arch, and great vessel pathology.

Which method is to be preferred in suspected cardiovascular injury depends on the human and material resources available within each institution. We believe that transoesophageal echocardiography, when available, should be considered first in cases of suspected traumatic cardiovascular lesions because of its safety, speed, accuracy, and convenience. Time from admission to diagnosis and surgery should be as short as possible in patients suffering cardiovascular injuries. In our institution transoesophageal echocardiography has become the preferred technique to achieve this objective with expert operators on call 24 hours a day.

\footnotetext{
1 Editorial. Traumatic injury of the heart. Lancet 1990; 336:1287-9.

2 LoCicero J, Mattox KL. Epidemiology of chest trauma. Surg Clin North Am 1989;69:15-19.
} 
3 Krasna MJ, Flancbaum MJ. Blunt cardiac trauma: clinical manifestations and management. Semin Thorac Cardiovasc Surg 1992;4:195-202.

4 Parmely LF, Mattingly TW, Manion WC, Jahnke EJ. Nonpenetrating traumatic injury of the aorta. Circulation 1958;17:1086-101

5 Akins CW, Buckley MJ, Daggen W, McIlduff JB, Austen WG. Acute traumatic disruption of the thoracic aorta: ten-year experience. Ann Thorac Surg 1981;31:305-9.

6 Plotnick GD, Hamilton S, Lee YC. The cardiologist and the trauma patient: noninvasive testing. Semin Thorac Cardiovasc Surg 1992;4:168-76.

7 Nelson JB, Bresticker MA, Nahrwood DL. Computed tomography in the initial evaluation of patients with blunt trauma. F Trauma 1992;33:722-7.

8 Miller FB, Richardson JD, Thomas HA, Cryer HM, Willing SJ. Role of CT in diagnosis of major arterial injury after blunt thoracic trauma. Surgery 1989;106: 596-603

9 Mirvis SE, Templeton PA. Imaging of thoracic trauma. Semin Thorac Cardiovasc Surg 1992;4:177-86.

10 Mirvis SE, Bidwell JK, Buddenmeyer EU, Diaconis JN, Pais SO, Whitley JE. Imaging diagnosis of traumatic aortic rupture: a review and experience at a major trauma center. Invest Radiol 1987;22:187-96.

11 Gundry SR, Williams S, Burney RE, MacKenzie JR, Cho $\mathrm{KJ}$. Indications for aortography: radiography after blunt chest trauma: a reassessment of the radiographic findings chest trauma: a reassessment of the radiographic findings associated with traum
Radiol 1983;18:230-7.

12 LaBerge JM, Jeffrey RB. Aortic lacerations: fatal complications of thoracic aortography. Radiology 1987;165:36-9.

13 Miller FA, Seward JB, Gersh BJ, Tajik AJ, Mucha P. Twodimensional echocardiographic findings in cardiac trauma. Am 7 Cardiol 1982;50:1022-7.

14 Helling TS, Duke P, Beggs CW, Crouse LJ. A prospective evaluation of 68 patients suffering blunt chest trauma for evidence of cardiac injury. F Trauma 1989;29:961-5.

15 Bu'Lock FA, Prothero A, Shaw C, et al. Cardiac involvement in seatbelt-related and direct sternal trauma: a prospective study and management implications. Eur Heart f 1994;15:1621-7.

16 Seward JB, Khandheria BK, Oh JK, et al. Transesophageal echocardiography: technique, anatomic correlations, implementations, and clinical applications. Mayo Clin Proc 1988;63:649-80.

17 Seward JB, Khandheria BK, Edwards WD, et al. Biplanar transesophageal echocardiography: anatomic correla-
tions, image orientation, and clinical applications. Mayo tions, image orientation, and

18 Baker SP, O'Neill B, Haddon W, Long WB. The Injury Severity Score: a method for describing patients with multiple injuries and evaluating emergency care. $\mathcal{F}$ Trauma 1974;14:187-96.

19 Copes WS, Champion HR, Sacco WJ, Lawnick MM, Keast SL, Bain LW. The Injury Severity Score revisited. $f$
Trauma 1988;28:69-77.

20 Tenzer ML. The spectrum of myocardial contusion. $f$ Trauma 1985;25:620.

21 Perry GJ, Helmcke F, Nanda NC, Byard C, Soto B Evaluation of aortic insufficiency by Doppler color flow imaging. F Am Coll Cardiol 1987;9:952-9.

22 Mohr-Kahaly S, Erbel R, Zenker G, et al. Semiquantitative grading of mitral regurgitation by color Doppler echocardiography. Int 7 Cardiol 1989;23:223-30.

23 Woodring JH, Dillon ML. Radiographic manifestations of mediastinal hemorrhage from blunt chest trauma. Ann Thorac Surg 1984;37:171-8.

24 Mirvis SE, Bidwell JK, Buddenmeyer EU, et al. Value of chest radiography in excluding traumatic aortic rupture. chest radiography in exclud

25 Burney RE, Gundry SR, MacKenzie JR, Whitehouse WM Wu SC. Chest roentgenograms in diagnosis of traumatic aortic rupture: observer variation in interpretation. Ches 1984;85:605-9.

26 Warren RL, Akins CW, Conn AKT, Hilgenberg AD, McCabe CJ. Acute traumatic disruption of the thoracic aorta: emergency department management. Ann Emerg Med 1992;21:391-6.

27 Daniel WG, Erbel R, Kasper W, et al. Safety of transesophageal echocardiography. A multicenter survey of 10,419 examinations. Circulation 1991;83:817-21.

28 Smith MD, Cassidy JM, Souther S, et al. Transesophageal echocardiography in the diagnosis of traumatic rupture of the aorta. N Engl f Med 1995;332:356-62.

29 Brooks SW, Young JC, Cmolik BL, et al. The use of transesophageal echocardiography in the evaluation of chest esophageal echocardiography in the

30 Shapiro MJ, Yanofsky SD, Trapp J, et al. Cardiovascular evaluation in blunt chest trauma using transesophageal echocardiography. $\mathcal{F}$ Trauma 1991;31:835-40.

31 de Belder A, Thomas M, Marrinan M. Traumatic rupture of the thoracic aorta diagnosed by transesophageal echocar diography. Br Heart $\mathcal{F}$ 1993; 70:393-4.

32 Dacosta A, Billard JL, Zeni F, et al. Rupture traumatique de l'isthme aortique diagnostiqee par l'echographie transoesophagienne. Arch Mal Coeur 1994;87:101-4.

33 Sandor $F$. Incidence and significance of traumatic mediastinal hematoma. Thorax 1967;22:43-62.

34 Rich NM. Injuries of the intrathoracic branches of the aortic arch. In: Rich NM, ed. Vascular trauma. Philadelphia: WB Saunders, 1978:287.

35 Woodring JH, Lee C, Jenkins K. Spinal fracture in blunt chest trauma. F Trauma 1988;28:789-93.

36 Davis GA, Sauerisen S, Chandrasekaran K, Karalis DG, Ross J, Mintz GS. Subclinical traumatic aortic injury diagnosed by transesophageal echocardiography. $A m$ Heart f 1992;123:534-6.

37 Khandheria BK, Oh JK. TEE: state of the art and future directions. Am $\mathcal{F}$ Cardiol 1992;69:61-75H.

38 Vlahakes GJ, Warren RL. Traumatic rupture of the aorta. $N$ Engl ₹ Med 1995;332:389-90. 